\title{
The Application of Federated Kalman Filtering in SINS/GPS/CNS Intergrated Navigation System
}

\author{
DENG Hong, LIU Guang-bin, CHEN Hao-ming, DENG Chun-lin
}

Research Inst. Of High-tech Honging Town 710025, Xi'an, China

\begin{abstract}
Federated filter was an important method to estimate high-precision navigation parameters based on "SINS/GPS/CNS". A no-feedback federated filter with UD_UKF algorithm was designed in the paper, a threetime amendment scheme to correct navigation parameters was designed at the same time and the mathematical model of SINS/GPS/CNS was established in launch inertial coordinate system too. The paper discussed the simulation conditions and a lot of simulations were carried out to compare 2 aspects: (1)the performance between four navigation mode, which respectively is SINS, SINS/GPS, SINS/CNS, SINS/GPS/CNS;(2)the estimate precision of federated filter and that of centralized Kalman filter. The results of simulation showed that the designed federated filter and amendment scheme based on SINS/GPS/CNS had high estimate precision and led to gain high hitting precision of ballistic missile, that is to say position errors were less than 20 meter and velocity errors were less than $0.1 \mathrm{~m} / \mathrm{s}$ in simulation.
\end{abstract}

Index Terms: Error correction; UD_UKF; no-feedback federated filter; "SINS/GPS/CNS" integrated guidance system

(C) 2012 Published by MECS Publisher. Selection and/or peer review under responsibility of the Research Association of Modern Education and Computer Science

\section{Introduction}

SINS/GPS/CNS integrated navigation system is the important way to improve the navigation precision of ballistic missile ${ }^{[1-3]}$. Although strap-down inertial navigation equipment (SINS) is the only navigation equipment which can give the estimations of velocity, position and attitude parameters of aerocraft meanwhile, guidance precision of SINS is decreased with the increase of time resulted from gyro drifts and accelerometer drifts. Thus, making use of GPS measurement to compensate the velocity and position error on the one hand, making use of CNS measurement to compensate the attitude error on the other is the way to improve navigation precision of SINS.

Data fusion process is an important issue in the SINS/GPS/CNS integrated navigation system. N.A. Carlson put forward federated filtering theory in 1988. The federated filtering precision is about equal to the traditional

* Corresponding author.

E-mail address: dhinsaec@163.com 
centralized Kalman filter, and has a better reliability[4-6]. At present, United States Air Force had appointed federated filter as universal filter in new-generation navigation system[4].

Firstly, a federated filter based on UD_UKF structure was designed in the background of ballistic missile in the paper. Then, mathematical model of SINS/GPS/CNS integrated navigation system was established in launch inertial coordinate system. Finally, simulation, comparing and analysis were carried out respectively in the background of SINS, SINS/GPS, SINS/CNS and SINS/GPS/CNS in the same parameter condition. The results showed the estimate precision of federated filter based on SINS/GPS/CNS was the most accurate system and it led to high navigation precision with efficiency and practicality.

\section{Federated Filter Design}

Federated filter includes six different structures[7]. Federated filter designed was based on the fourth structure with "no-feedback" to satisfy fault tolerant ability, which composed by two sub-filters and one main-filter with the reference was SINS shown in figure 1. The two sub-filters respectively were SINS/CNS and SINS/GPS. Moreover, Unscented Kalman Filter (UKF) is one of the new style filters based on unscented transform (UT). It avoids linearization error and calculation of Jacobian matrix which extended Kalman filter EKF has to face. And its state estimate accuracy is higher than that of EKF. Thus, UKF was adopted in simulation. But, calculation error in SINS/CNS sub-filter conflicted with nonnegative constraints during UKF filter process. Thus, UD_UKF based on UDUT algorithm was adopted in the SINS/CNS system.

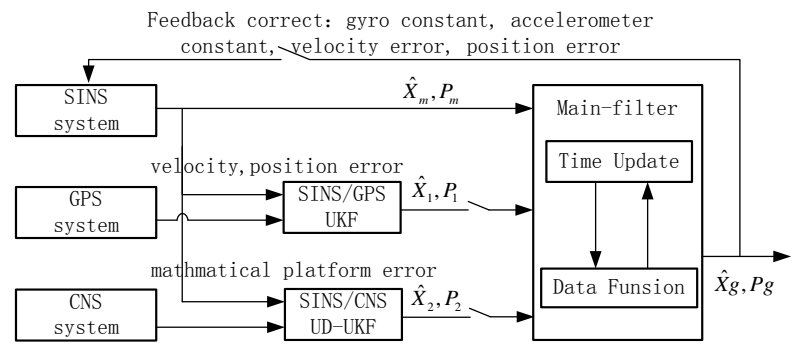

Fig. 1 Federated Filter Structure based on SINS/GPS/CNS

Main-filter did not carry out measurement update. And data fusion of SINS, SINS/CNS, and SINS/GPS were implemented with formula 1 as follows after time update.

$\left\{\begin{array}{c}P_{g}^{-1}=P_{1}^{-1}+P_{2}^{-1}+P_{m}^{-1} \\ \hat{X}_{g}=P_{g}\left(P_{1}^{-1} \hat{X}_{1}+P_{2}^{-1} \hat{X}_{2}+P_{m}^{-1} \hat{X}_{m}\right)\end{array}\right.$

This includes: $\hat{X}_{g}, P_{g}$ respectively were the optimal estimated value and the optimal covariance of mainfilter. $\hat{X}_{i}, P_{i}(i=1,2, m)$ were the optimal estimated value sequences and the optimal covariance sequences of sub-filter.

\section{The Mathematical Model of Integrated Navigation System ${ }^{[8-9]}$}

\section{A. State Equation}

In order to obtain high estimate precision of ballistic missile, constant drift and first-order drift will often be taken into account in the state equation at the same time. Therefore, the state equation was showed as below with 15-dimensional system state variable was taken into account.

$$
x=\left[\begin{array}{lllll}
\phi & \delta V & \delta P & \varepsilon & \nabla
\end{array}\right]^{T}
$$




$$
\dot{X}(t)=F(t) X(t)+G(t) W(t)
$$

The system state variable includes: three mathematical misalignment angles, three velocity errors, three position errors, three gyro constant drifts and three accelerometer constant drifts. The system noise $W(t)$ includes: three gyro white Gaussian noise, three accelerometer white Gaussian noise. The form of state matrix was as follows.

$$
F(t)=\left[\begin{array}{ccccc}
0_{3 \times 3} & 0_{3 \times 3} & 0_{3 \times 3} & C_{b}^{f} & 0_{3 \times 3} \\
F_{b} & 0_{3 \times 3} & F_{a} & 0_{3 \times 3} & C_{b}^{f} \\
0_{3 \times 3} & I_{3 \times 3} & 0_{3 \times 3} & 0_{3 \times 3} & 0_{3 \times 3} \\
0_{3 \times 3} & 0_{3 \times 3} & 0_{3 \times 3} & 0_{3 \times 3} & 0_{3 \times 3} \\
0_{3 \times 3} & 0_{3 \times 3} & 0_{3 \times 3} & 0_{3 \times 3} & 0_{3 \times 3}
\end{array}\right] \quad G(t)=\left[\begin{array}{cc}
C_{b}^{f} & 0_{3 \times 3} \\
0_{3 \times 3} & C_{b}^{f} \\
0_{3 \times 3} & 0_{3 \times 3} \\
0_{3 \times 3} & 0_{3 \times 3} \\
0_{3 \times 3} & 0_{3 \times 3}
\end{array}\right]
$$

This includes: $F_{a}$ was first-partial derivative of acceleration of gravity with respect to position coordinate, $F_{b}$ was Skew-Symmetric Matrix consisting of the apparent acceleration, $C_{b}^{f}$ was the transformation matrix from the body coordinate system to the inertial coordinate system.

The result of discreting the state equation according to formula 7 was as follows:

$$
\begin{aligned}
& \Delta X_{k}=\Phi_{k \mid k-1} X_{k-1}+G_{k-1} W_{k-1} \\
& \left\{\begin{array}{l}
\Phi_{k \mid k-1}=I+F_{k-1} \Delta T+\frac{1}{2 !} F_{k-1}^{2} \Delta T^{2}++\frac{1}{3 !} F_{k-1}^{3} \Delta T^{3}+\ldots \\
G_{k-1}=\Delta T\left(1+\frac{1}{2 !} F_{k-1} \Delta T+\frac{1}{3 !} F_{k-1}^{2} \Delta T^{2}+\ldots\right) G_{k-1}
\end{array}\right.
\end{aligned}
$$

This includes: $\Phi_{k \mid k-1}$ was the one-step transition matrix, $G_{k-1}$ was noise matrix, $W_{k-1}$ was white noise sequence with zero mean, and $\Delta T$ was filter step.

\section{B. Measuring Equations}

\section{1) SINS/GPS Measuring Equation}

The measuring equation of SINS/GPS was as follows:

$$
\begin{aligned}
& Z_{1}(t)=H_{1}(t) X(t)+V_{1}(t) \\
& H_{1}(t)=\left[\begin{array}{lll}
0_{6 \times 3} & I_{6 \times 6} & 0_{6 \times 3}
\end{array}\right]
\end{aligned}
$$

This includes: ${ }^{V_{1}(t)}$ was white noise with zero mean of GPS navigation system.

\section{2) SINS/CNS Measuring Equation}

The measuring equation of SINS/CNS was as follows:

$$
Z_{2}(t)=\left[\begin{array}{ll}
I_{3 \times 3} & 0_{3 \times 2}
\end{array}\right] X(t)+V_{2}(t)
$$

This includes: $V_{2}(t)$ was white noise with zero mean of CNS navigation system. The attitude error of SINS and CNS can be gained by the measurement of SINS/CNS sub-filtering system, but only mathematical misalignment angles can be applied to SINS/GPS/CNS integrated navigation system. Thus, the attitude angle error must be converted into misalignment angle to engage in UD_UKF filter. The form of transform matrix between misalignment angle and attitude angle error was as follows. (Derivative process was not discussed in the paper). In additional, quaternion was adopted in the attitude express of SINS/GPS/CNS integrated navigation system because of not involving operations of triangle, no oddity, and less calculation. 


$$
\begin{aligned}
& {\left[\begin{array}{l}
\delta q_{0} \\
\delta q_{1} \\
\delta q_{2} \\
\delta q_{3}
\end{array}\right]=\left[\begin{array}{c}
\delta q_{0 c n s}-\delta q_{0 \sin s} \\
\delta q_{1 c n s}-\delta q_{1 \sin s} \\
\delta q_{2 c n s}-\delta q_{2 \sin s} \\
\delta q_{3 c n s}-\delta q_{3 \sin s}
\end{array}\right]=\frac{1}{2}\left[\begin{array}{ccc}
\hat{q}_{3} & \hat{q}_{2} & \hat{q}_{1} \\
\hat{q}_{2} & -\hat{q}_{3} & -\hat{q}_{0} \\
-\hat{q}_{1} & -\hat{q}_{0} & \hat{q}_{3} \\
-\hat{q}_{0} & \hat{q}_{1} & -\hat{q}_{2}
\end{array}\right]\left[\begin{array}{l}
\phi_{x} \\
\phi_{y} \\
\phi_{z}
\end{array}\right]} \\
& {\left[\begin{array}{l}
\phi_{x} \\
\phi_{y} \\
\phi_{z}
\end{array}\right]=-\frac{2}{\hat{q}_{0}}\left[\begin{array}{ccc}
\hat{q}_{1} \hat{q}_{3}-\hat{q}_{0} \hat{q}_{2} & \hat{q}_{0} \hat{q}_{1}+\hat{q}_{2} \hat{q}_{3} & \hat{q}_{0}^{2}+\hat{q}_{3}^{2} \\
\hat{q}_{2}+\hat{q}_{0} \hat{q}_{3} & \hat{q}_{0}^{2}+\hat{q}_{2}^{2} & \hat{q}_{2} \hat{q}_{3}-\hat{q}_{0} \hat{q}_{1} \\
\hat{q}_{0}^{2}+\hat{q}_{1}^{2} & \hat{q}_{1} \hat{q}_{2}-\hat{q}_{0} \hat{q}_{3} & \hat{q}_{0} \hat{q}_{2}+\hat{q}_{1} \hat{q}_{3}
\end{array}\right]\left[\begin{array}{c}
\delta q_{1} \\
\delta q_{2} \\
\delta q_{3}
\end{array}\right]}
\end{aligned}
$$

There had one constraint condition which was as shown in formula 13.

$$
q_{0} \delta q_{0}+q_{1} \delta q_{1}+q_{2} \delta q_{2}+q_{3} \delta q_{3}=0
$$

\section{Simulation and Result Analyses}

To verify the performance of federated filter designed in paper based on SINS/GPS/CNS, a lot of experiments were carried out with 2 aspects: navigation mode and two filter methods. Simulation condition and results were discussed as follows. In additional, to gain better navigation precision, three-time amendment was designed during the federated filter process shown in figure 2.

\section{Simulation Condition}

Simulation condition included three aspects.

\section{1) Missile trajectory of simulation}

Missile trajectory established in the simulation in the background that the earth was LAG-75 ellipsoid and taking strap-down ballistic missile as an example. A dotted line was expressed true trajectory in simulation.

Supposed the missile was Vertical launched that pitch is equal to 90 degree with initial position $P 0$ and initial velocity $V 0$ were both equal to zero and initial misalignment angle was equal to $\left[\begin{array}{lll}-10 & 10 & 80\end{array}\right.$ in the launching coordinate system.

\section{2) Simulation parameters}

Main simulation parameters were listed in table 1 and all data unit involved in the simulation procedure were

\begin{tabular}{|c|c|}
\hline Name & Parameters \\
\hline Gyro constant drift & $\begin{array}{l}\varepsilon=\left[\begin{array}{lll}0.15 & 0.15 & 0.15\end{array}\right] \\
\left({ }^{0} / \mathrm{h}\right)\end{array}$ \\
\hline Accelerometer constant drift & $\nabla=\left[\begin{array}{lll}20 & 20 & 20\end{array}\right]$ \\
\hline GPS velocity error & $\delta V=\left[\begin{array}{lll}0.2 & 0.2 & 0.2\end{array}\right] \quad(\mathrm{m} / \mathrm{s})$ \\
\hline GPS position error & $\delta P=\left[\begin{array}{lll}20 & 20 & 20\end{array}\right]$ \\
\hline CNS attitude measurement error & $\delta \theta=\left[\begin{array}{lll}10 & 10 & 10\end{array}\right]$ \\
\hline
\end{tabular}
according to standard units such as: $\mathrm{m}, \mathrm{s}$, rad and etc..

TABLE I. SIMULATION PARAMETERS

\section{3) Amendment scheme}

Supposed simulation started to be 80 second after primary engine shut down, which supposed that pitch was 42.6 degrees, yaw and roll both were 0 degree. Filter period was 20 min, sampling interval was 1 second, and fusion interval was 1 second. The time of three-time amendment respectively was 400 second, 800 second and 1100 second. 


\section{Simulation Results}

Simulation for comparing the navigation performance between SINS, SINS/GPS, SINS/CNS and SINS/GPS/CNS was carried out using Matlab with Monte Carlo method. A branched line was expressed trajectory of SINS in simulation and a bold line was expressed trajectory of SINS/GPS/CNS in simulation.

It can be seen from figure 2 that trajectory of SINS/GPS/CNS in simulation is very close to true trajectory in simulation in three-dimensional space. The navigation precision was improved due to gyro constant drift and accelerometer constant drift were estimated in high precision on one hand and velocity and position parameters were compensated three times in simulation on the other.

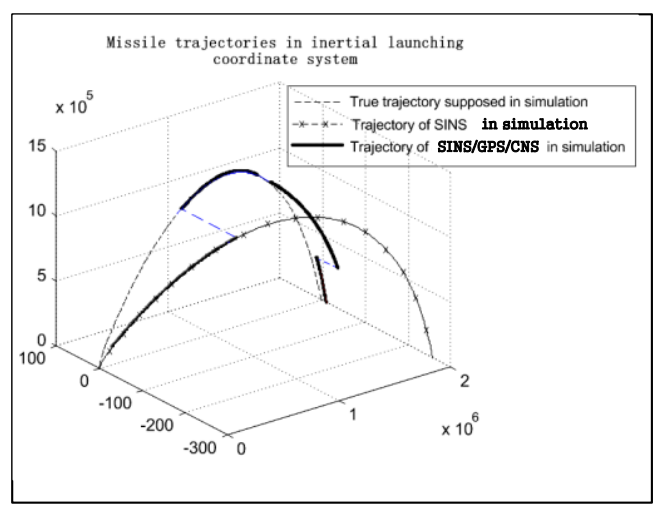

Fig. 2 Trajectories of missile in simulation

The performance of federated filter based on SINS/GPS/CNS was analyzed in detail as follows.

(1)It can be seen from the figure 3(a) (b) (c) that three-axis velocity error and position error propagation had divergent tendency based on SINS/CNS, and accelerometer constant drift couldn't be estimated effective. The reasons analysis of the case was: velocity error and position error, which used to compensate the velocity and position parameters, couldn't be estimated based on SINS/CNS due to CNS navigation system can only give attitude measurements.
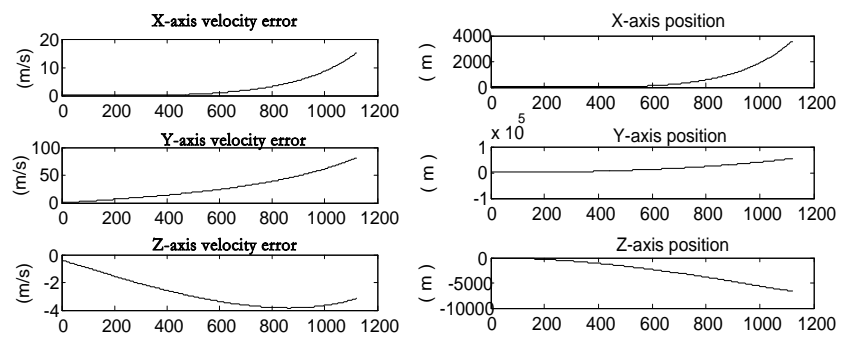

(a) Three-axis Velocity Error

(b) Three-axis Position

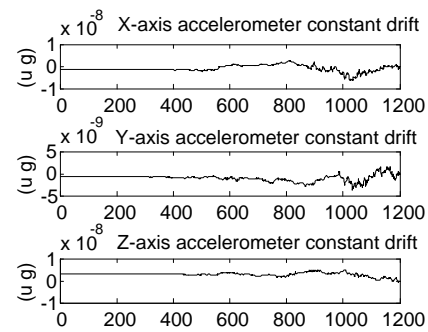

(c) Three-axis Accelerometer Constant Error

Fig. 3 Error estimated in federated filter based on SINS/CNS 
(2) It can be seen from the figure 4 that the mathematical misalignment angles and gyro constant drift couldn't be estimated effective based on SINS/GPS. The reasons analysis of the case was GPS navigation system can not give attitude measurement at all.

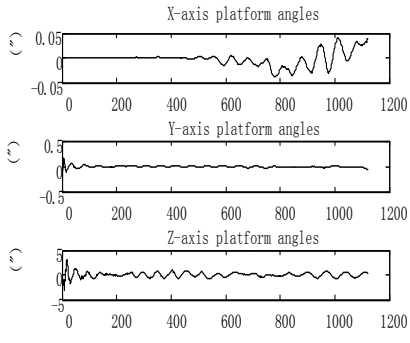

(a) Three-axis platform angles

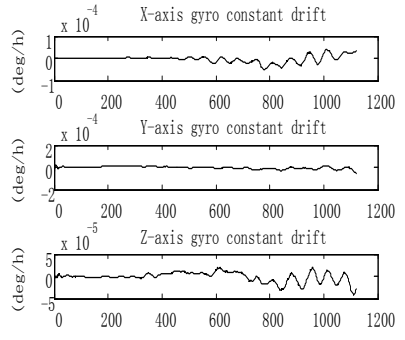

(b) Three-axis gyro constant drift

Fig. 4 Error estimated in federate filter based on SINS/GPS

(3)The statistical data resulted from 200-time simulations showed the values of gyro constant drift and the values of accelerometer constant drift in federate filter based on SINS/GPS/CNS. This includes: black line was expressed centralized Kalman filter of SINS/GPS/CNS; red line was expressed federate filter of SINS/GPS/CNS. Analysis can be drawn: [1] All state variables of SINS/GPS/CNS can be estimated in high precision by velocity, position, attitude measurements. [2] It was shown from figure 5(a)-(d) that the performance of federate filter is very close to the performance of centralized Kalman filter. [3] It was shown from figure 5(e)-(f) that position error was less than 20 meter and velocity error was $0.1 \mathrm{~m} / \mathrm{s}$. The navigation precision was improved apparently.

TABLE II. ANALYSIS OF NAVIGATION PARAMETERS

\begin{tabular}{c|ccc}
\hline Name & $\begin{array}{c}\mathrm{x}- \\
\text { axis }\end{array}$ & $\begin{array}{c}\mathrm{y}- \\
\text { axis }\end{array}$ & $\mathrm{z}$-axis \\
\hline $\begin{array}{c}\text { Gyro constant error }\left({ }^{0} / \mathrm{h}\right) \\
\text { Accelerometer constant } \\
\text { error }(\mu \mathrm{g})\end{array}$ & 0.004 & 0.004 & 0.004 \\
$\begin{array}{c}\text { Initial misalignment angles } \\
(\mathrm{\prime})\end{array}$ & 0.6 & 0.8 & 0.5 \\
\hline
\end{tabular}
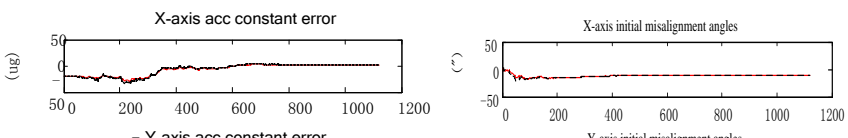

可
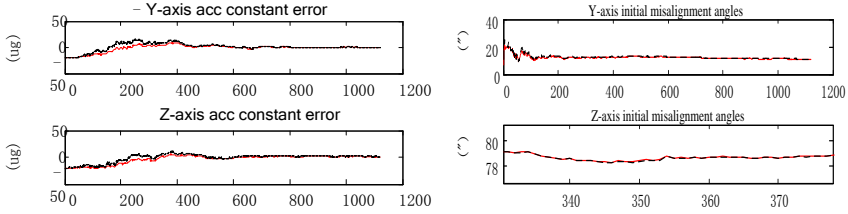

(a)Three-axis acc constant error

(b)Three-axis initial misalignment angles 

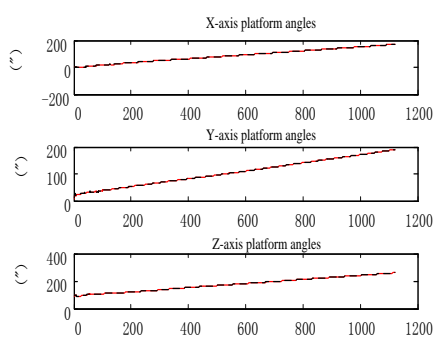

(c) Three-axis platform angles
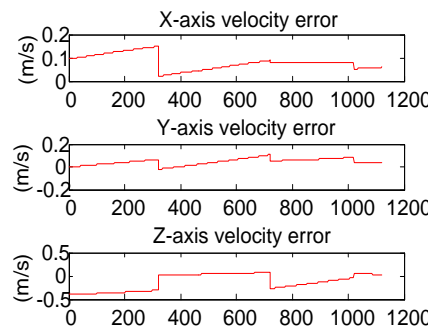

(e)Three-axis velocity error
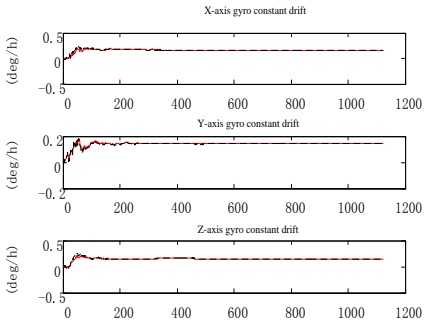

(d) Three-axis gyro constant drift
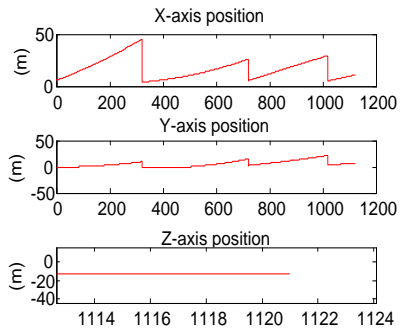

(f) Three-axis position estimation

Fig. 5 Navigation parameters estimated based on SINS/GPS/CNS

\section{Conclusion}

SINS/GPS/CNS integrated guidance system had complementary advantages. The hitting precision of ballistic missile was improved based on SINS/GPS/CNS by reasonable amendment scheme and high-precision estimation of navigation parameters, such as velocity error, position error, gyro constant drift, accelerometer constant drift and platform drift. The simulation results showed that the estimate precision of accelerometer error, accelerometer constant drift and gyro error based on SINS/GPS/CNS was close to the precision based on SINS/GPS and the estimate precision of mathematical misalignment angles and gyro constant drift based on SINS/GPS/CNS was close to the precision based on SINS/CNS. In additional, SINS/GPS/CNS integrated system allowed less accuracy of strap-down inertial guidance measuring equipment in comparison with SINS and was easy to be realized in project. It is worthwhile to note that improving of the amendment scheme and shorting the time of convergence both are worthy of further study to improve navigation precision further.

\section{References}

[1] WANG Xin-guo, LI Ai-hua, XU Hua-long. Data Fusion of SINS/CNS/GPS in Application of Missile Guidance Syste[J]. Journal of System Simulation. 2009,5(21):3062 3070 (In Chinese)

[2] WANG Hai-bo, CHAI Ying-bo. Position and attitude integrated method for INS/GPS/CNS integrated systems based on federated filter [J]. Journal of Chinese Inertial Technology.2008,4(16):196 199 (In Chinese)

[3] WANG Xin-long, XIE Jia, GUO Long-hua. A Study on Model of Strapdown Inertial/Starlight Integrated Guidance System for Ballistic Missiles [J]. Journal of Ballistics.2008, Vol. (20):87 91 (In Chinese)

[4] Carlson N A. Federated filter for fault-torlerant integrated navigation systems[A]. PLANS[C], 1988:110119

[5] Carlson N A. Federated filter for decentraliezed parallel processes[J]. IEEE AES. 1990, 26(3):517-525. 
[6] Carlson,N.A. and Beraducci, M.P.. Federated Kalman filter simulation results[J]. Navigation Fall. 1994, Vol.(41)3:297 321.

[7] Qin Yong-yuan, Zhang Hong-yue, Wang Shu-hua. Kalman filtering and navigation principles [M].Northwestern Polytechnical University Press.2004,7 (In Chinese)

[8] Shen Gong-xun, Sun Yuan-feng. Information fusion theory in the INS/CNS/GPS Integrated Navigation System [M]. National Defence Industrial Press,1998 (In Chinese)

[9] Fang Jian-cheng, Ning Xiao-ning.celestial navigation principle and application[M]. Beijing University of Aeronautics and Astronautics Publishing House press,2006 (In Chinese) 